Research Paper

\title{
Regulatory and Effector Helper T-Cell Profile after Nerve Xenografting in the Toll-Like Receptor-Deficient Mice
}

\author{
Cheng-Shyuan Rau1*; Ming-Wei Lin ${ }^{2 *}$; Shao-Chun $\mathrm{Wu}^{3^{*}}$; Yi-Chan $\mathrm{Wu}^{2}$, Tsu-Hsiang $\mathrm{Lu}^{2}$, Siou-Ling Tzeng ${ }^{2}$, \\ Yi-Chun Chen ${ }^{2}$, Chia-Jung Wu ${ }^{2}$, Ching-Hua Hsieh ${ }^{2}{ }^{\circledR}$ \\ 1. Department of Neurosurgery, Kaohsiung Chang Gung Memorial Hospital and Chang Gung University College of Medicine, Taiwan \\ 2. Department of Plastic and Reconstructive Surgery, Kaohsiung Chang Gung Memorial Hospital and Chang Gung University College of Medicine, \\ Taiwan \\ 3. Department of Anesthesiology, Kaohsiung Chang Gung Memorial Hospital and Chang Gung University College of Medicine, Taiwan
}

"These authors contributed equally to this work

$\square$ Corresponding author: Ching-Hua Hsieh, M.D., PhD., FACS, Department of Plastic and Reconstructive Surgery Kaohsiung Chang Gung Memorial Hospital and Chang Gung University College of Medicine, No.123, Ta-Pei Road, Niao-Song District, Kaohsiung City 833, Taiwan. Tel: 886-7-7327476; E-mail: m93chinghua@gmail.com

() 2015 Ivyspring International Publisher. Reproduction is permitted for personal, noncommercial use, provided that the article is in whole, unmodified, and properly cited. See http:/ /ivyspring.com/terms for terms and conditions.

Received: 2015.04.01; Accepted: 2015.07.18; Published: 2015.08.01

\begin{abstract}
Introduction: The balance between regulatory T cells (Tregs) and effector T help cells (Th cells) is critical for the control of adaptive immune response during nerve transplantation. However, whether the homeostasis of immune regulation between Tregs and Th cells requires toll-like receptor (TLR) signaling is unclear. The aim of this study is to profile the distribution of spleen Tregs and Th cells in a mouse model of nerve xenografting in the TLR2 and NF-KB gene knockout mice.

Methods: The sciatic nerve was taken from a SD rat or an allogeneic mouse and transplanted to a right back leg of recipient C57BL/6, TLR2 ${ }^{-1-}$, or NF- $\mathrm{KB}^{-1-}$ mice by subcutaneous transplantation. After 7 days, the $T$ lymphocytes were then isolated from spleen, stained with phenotyping kits, and analyzed by flow cytometry.

Results: The results showed that Tregs were decreased after nerve xenografting in the recipient C57BL/ 6 mouse. In addition, nerve xenografting also increased the Th1 and Th17 but not the Th2 cell populations. In contrast, amelioration of the Tregs elimination was found in TLR2 $2^{-1-}$ and $\mathrm{NF}-\mathrm{KB}^{-1-}$ mice after transplantation of the nerve xenograft. Moreover, the mice lacking TLR2 or NF-KB showed attenuation of the increase in Thl and Th17 cells after nerve xenografting.

Conclusions: TLR signaling is involved in T cell population regulation during tissue transplantation. Knock-out of TLR2 and NF-KB prevented Tregs elimination and inhibited Th1- and Th17-driven immune response after nerve xenografting. This study highlighted the potential of inhibiting TLR signaling to modulate $T$ cell-mediated immune regulation to facilitate tolerance to nerve transplantation.
\end{abstract}

Key words: Nerve xenografting, Toll-like receptor (TLR), Regulatory T cells (Tregs), T help cells (Th cells)

\section{Introduction}

Nerve allograft had been reported to successfully treat the gap of the injured peripheral nerve. However, neurologic recovery with nerve allotransplantation is still limited by immune response over-activation and graft rejection and requires a short-term use of immunosuppressive agents. Nerve allo- or xeno-transplantation results in activation of both innate and adaptive immunity. TLRs are sensors 
of both innate and adaptive immunity, and they play critical roles in nerve graft rejection and dysfunction after transplantation [1, 2]. Moreover, pathogens, surgical trauma and ischemic injury in the graft may also result in TLR stimulation [3]. Activation of TLRs may initiate intracellular signal transduction and lead to activation of the transcription factor, NF- $\mathrm{kB}$, to release proinflammatory cytokines, such as IL-1 $\beta$, TNF- $\alpha$, and IL17 $[4,5]$.

$\mathrm{CD}^{+}$regulatory $\mathrm{T}$ cells (Tregs) expressing FOXP3 play a critical role in the maintenance of immune tolerance during tissue transplantation [6]. The presence of Tregs is associated with allograft survival [7-9]. However, other phenotypes of $\mathrm{T}$ lymphocytes such effector $\mathrm{T}$ helper cells including $\mathrm{CD} 4^{+} \mathrm{INF} \gamma^{+} \mathrm{Th} 1$, $\mathrm{CD}^{+}{ }^{+} \mathrm{IL} 4^{+} \mathrm{Th} 2$, and CD4+IL17 ${ }^{+}$Th17 cells exert deleterious effects on tissue by activating of dendritic cells macrophages, and antigen-presenting cells. They secrete cytokines to activate inflammatory pathways, mainly through macrophage activation. However, overactivation of either pathway may cause tissue damage and result in graft rejection [10-12]. Tregs can regulate and suppress Th cells function mainly by cell-to-cell contact-dependent and antigen-independent mechanisms [13, 14]. Therefore, prolonged allograft acceptance requires Tregs, and the balance between Tregs and Th cells is critical for the inhibition of autoimmunity and the magnitude of the adaptive immune response [10, 15].

A previous study demonstrated that TLRs play a role in the maintenance of Tregs, are involved in $\mathrm{T}$ cell development [16]. Activation of TLRs can reprogram naïve $\mathrm{T}$ cells or Tregs to become effector Th cells [2, 17]. Therefore, TLR 2 and its downstream target, $\mathrm{NF}-\mathrm{kB}$, may play a role in the homeostasis of immune regulation between Tregs and Th cells. Moreover, nerve xenografting had been reported to induce more severe immunoresponse of the recipient than the nerve allografting. Therefore, in this study, the aim is to investigate the role of TLR2/NF-KB on the homeostasis of immune regulation between Tregs and Th cells by profiling their distribution in the spleen in a mouse model of nerve xenografting using the TLR2 and NF-kB gene knockout mice.

\section{Methods}

\section{Animal experiments}

C57BL/ 6 mice and SD rats were purchased from BioLasco (Taipei, Taiwan). Tlr2-/- (B6.129-Tlr2tm1Kir/ $\mathrm{J})$, and NF-kB-/- (B6.Cg-Nfkb1tm1Bal/J) mice were purchased from Jackson Laboratory (Bar Harbor, USA). All housing conditions were maintained, and surgical procedures, including analgesia, were performed in an Association for Assessment and Ac- creditation of Laboratory Animal Care International-accredited SPF facility according to national and institutional guidelines. Animal protocols (permission number No. 2012091304) were approved by Chang Gung Memorial Hospital. Briefly, mice or rats were anesthetized with a combination of ketamine and xylazine, and the right back leg incision was made. The sciatic nerve $(1 \mathrm{~cm})$ was taken from a SD rat and transplanted to a right back leg of recipient mice by subcutaneous transplantation. This type of transplantation is defined as xenograft. The donor sciatic nerve taken from the same species of mouse is defined as allograft. The mice were sacrificed after 7 days after the surgery, and the spleen was removed for the $T$ lymphocytes isolation.

\section{T lymphocyte isolation and flow cytometry analysis}

The spleen was removed from the mice and dissected. Splenic cell suspensions were gently pressed through a sterile $100 \mu \mathrm{m}$ nylon mesh, and lymphocytes were isolated by ficoll gradient centrifugation (GE Healthcare, Sweden). The isolated lymphocytes were stained with a mouse Th1/Th2/Th17 phenotyping kit (the fluorescent antibodies: CD4, IFN- $\gamma$, IL-4 or IL-17A for detecting Th1, Th2 or Th17) and a mouse Th17/Treg phenotyping kit (the fluorescent antibodies: Foxp3 and CD4 for detecting Tregs) (BD Pharmingen, USA), and were acquired in a BD LSR II flow cytometer (BD Biosceinces, USA).

\section{Statistical analysis}

The data collected were analyzed using SPSS v.20 statistical software (IBM, Armonk, NY) for the independent Student's t-tests. All results are presented as the mean \pm standard error. A p-value less than 0.05 was considered statistically significant.

\section{Results}

\section{Nerve xenografting decreased Tregs population in wild type mice but not in TRL2-- or NF-KB-/- mice}

To determine whether TLR2 or NF- $\mathrm{kB}$ plays a role in Tregs population modulation in nerve transplantation, we isolated spleen $\mathrm{T}$ lymphocytes from wild type or TLR2 $\%$ or $\mathrm{NF}-\mathrm{KB}^{-} /$mice to analyze $\mathrm{CD}^{+}{ }^{+} \mathrm{Foxp}^{+}{ }^{+}$Tregs population in nerve allografts or xenografts. By flow cytometry analysis, we found that the $\mathrm{CD}^{+}{ }^{+} \mathrm{Foxp}^{+}$Tregs population was decreased in the spleen of control wild-type mice after nerve xenografting compared to that after allografting. However, elimination of the Tregs population was ameliorated in NF-kB-/- mice (Fig 1). The results indicate that NF-kB may participate in Treg-mediated immune suppression after nerve xenografting. 
A

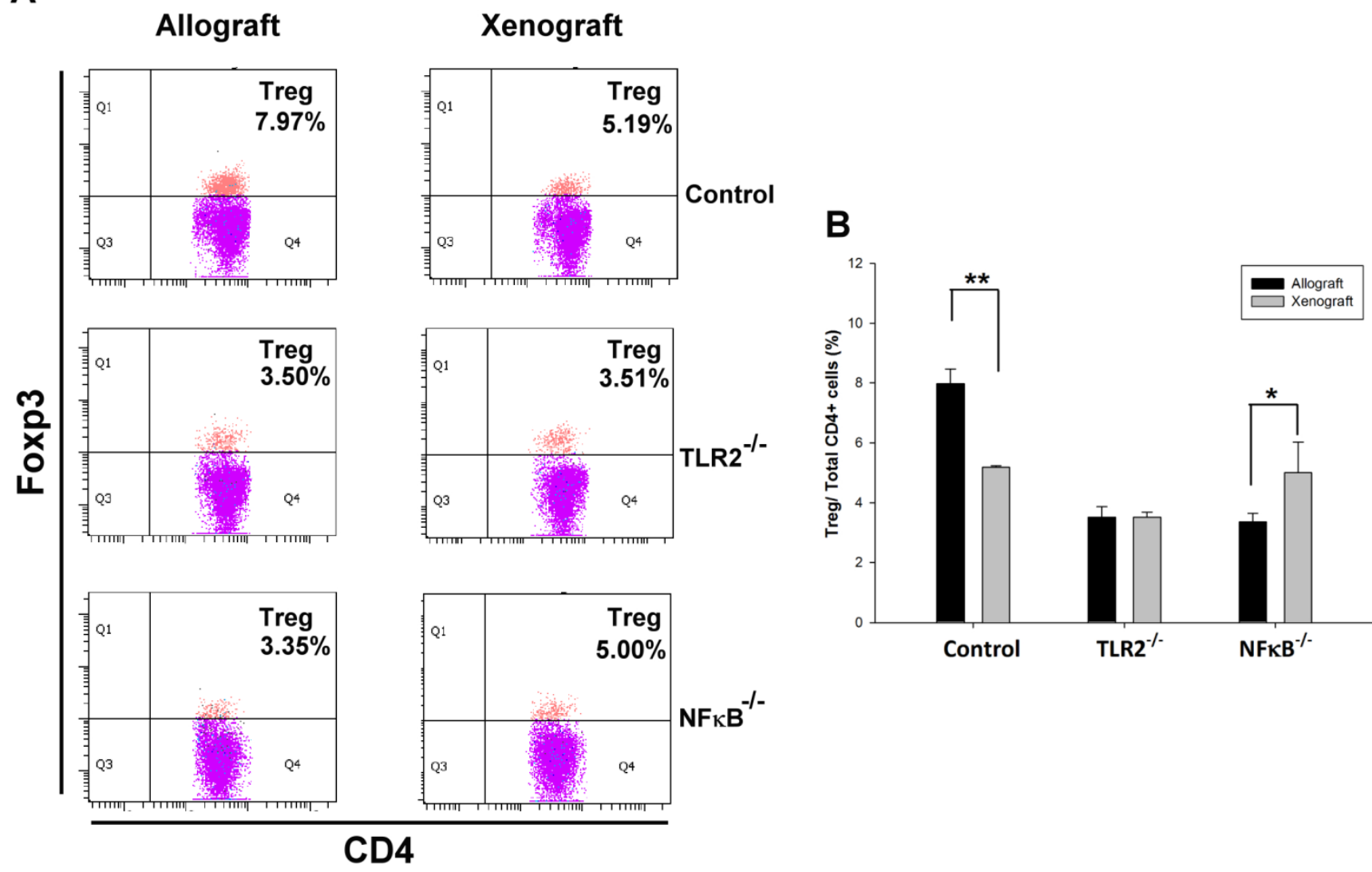

Figure 1. Knock-out of TLR2 and NF-KB prevented Tregs elimination after nerve xenografting. (A) Flow cytometry analysis of CD4 $4^{+}$Foxp $3^{+}$ Tregs population in nerve allografts or xenografts. (B) Quantification of the $C D 4^{+} F o x p 3^{+}$Tregs population from flow cytometry analysis. The $C D 4^{+} \mathrm{Foxp} 3^{+}$ Tregs population was decreased after nerve xenografting compared to allografting. Data are representative of 3 experiments with similar results. $(n=3-5$, $*_{p}<0.05$ and $*^{*} p<0.01$ vs corresponding allograft).

\section{Knock-out of TLR2 or NF-KB decreased Th 1 but not Th2 cells after nerve xenografting}

To determine whether TLR2 or NF-kB plays a role in Th1 or Th2 population regulation after nerve xenografting, we analyzed the Th1 or Th2 population after nerve xenografting compared to allografts in wild-type, TLR2 $\%$ - and NF-kB $\%$ mice. The flow cytometry analysis results indicate that knock-out of TLR2 or NF-kB decreased of Th1 but not Th2 population relative to the populations in control wild-type mice (Fig 2).

\section{Knock-out of TLR2 or NF-KB inhibited the Th 17 population increase after nerve xenografting}

To determine whether the Th17 population is involved in TLR2- or NF-kB-dependent pathways, we analyzed Th17 cell population in wild-type, TLR2-/-, or NF-kB-/- mice after nerve xenografting compared to allografting. We found that the Th17 population elevation was inhibited after nerve xenografting in TLR2 $-/-$ and NF-kB-/- mice (Fig 3). TLR2 and NF-kB may play a role in Th17-medated immune response after nerve xenografting.

\section{Treg/(Th1+Th17) balance is TLR2 or NF-KB dependent after nerve xenografting}

The balance between Tregs and Th cells is critical for the inhibition of autoimmunity. The results of the flow cytometry analysis showed that balance of the Tregs population over the Th1 and Th17 population was disturbed in wild-type mice after nerve xenografting. The value of Treg/(Th1+Th17) was decreased in control wild type but not in TLR2 $\%$ or NF-kB $/$ - mice (Fig 4).

\section{Discussion}

Successful transplantation depends on the modulation of adaptive immunity in graft transplantation, as previous studies demonstrated that $\mathrm{T}$ cells are necessary and sufficient to reject almost all allogeneic tissues [18]. Tregs function as immune suppressors in regulating effector Th cells [13]. Therefore, the balance between Tregs and Th cells is critical for inhibition of the autoimmune response and graft rejection. 


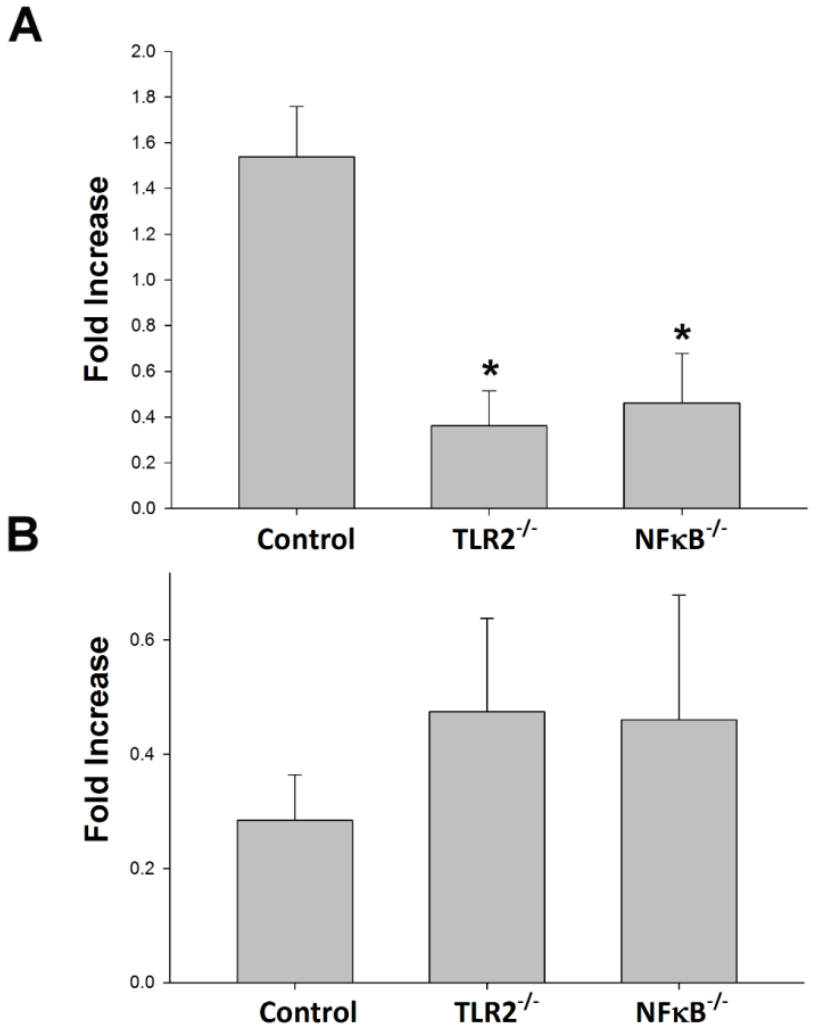

Figure 2. Knock-out of TLR2 and NF-KB prevented Th1 but not Th2 elevation after nerve xenografting. (A) Th1 and (B) Th2 cell expression in wild type or TLR2 ${ }^{-/-}$or NF- $\mathrm{KB}^{-/-}$mice in xenografts compared to allografts by flow cytometry analysis. Data are expressed as fold increases (mean $\pm S D)$ and are representative of 3 experiments with similar results. ( $n=3-5, *_{p}<0.05$ vs control wild-type mice).

A

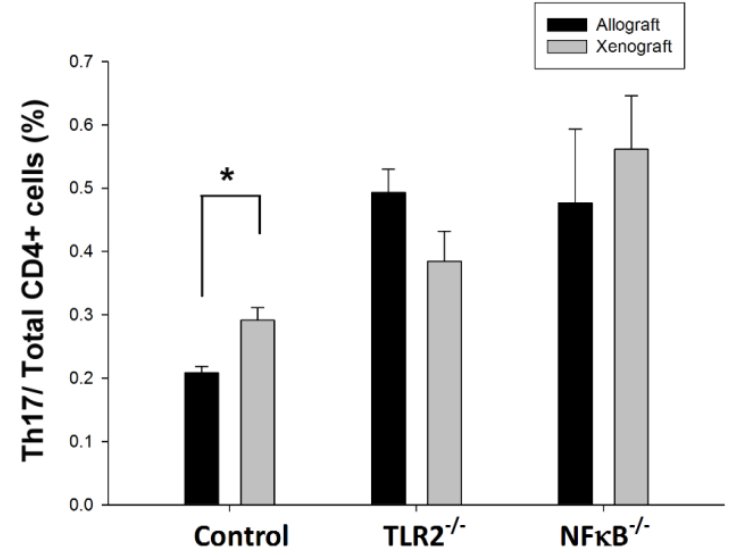

Figure 3. Knock-out of TLR2 and NF-KB inhibited Th17 increase after nerve xenografting. The Th17 cell population was increased after nerve xenografting compared to allografts in wild-type but not in TLR2 $2^{-1}$ or $\mathrm{NF}-\mathrm{kB}^{-1-}$ mice, as observed by flow cytometry analysis. Data are presented as the mean $\pm S D$ and are representative of 3 experiments with similar results. ( $n=3-5,{ }^{*} p<0.05$ vs control wild type mice).

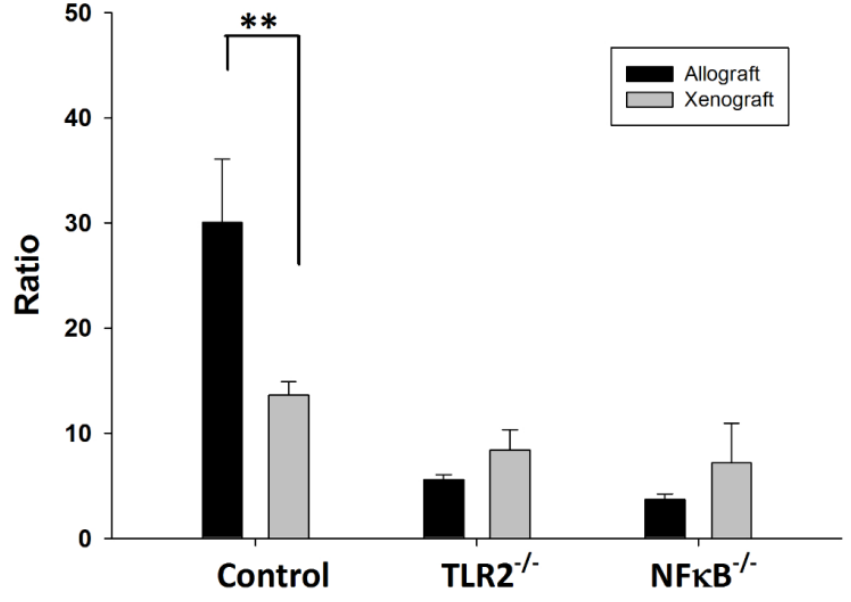

Figure 4. Ratio of Treg/(Th1+Th17) in wild type, $\mathrm{TLR2}^{-1}$, NF-KB ${ }^{-1-}$ mice after nerve xenografting. The value of Treg/(Th1+Th17) was decreased in control wild type but not in TLR2 ${ }^{-/}$or NF-kB ${ }^{-/-}$mice. Data are presented as the mean $\pm S D$. $\left(n=3,{ }^{*}<<0.05\right.$ vs control wild-type mice).

TLRs are the first-line sensor for innate immunity, and mounting evidence suggests that stimulation of TLR activated serious inflammatory responses which resulted in tissue injury and graft rejection after transplantation $[1,18,19]$. Although TLRs and T cells are known to play major roles in innate immunity, whether knock-out of TLRs can regulate the $\mathrm{T}$ cell population after tissue transplantation remains unknown. To determine whether TLR2 is important in the modulation of Tregs and Th cells in tissue transplantation, we used a model of nerve transplantation in TLR2 or NF-KB knock-out mice. Our study demonstrated that knock-out of TLR2 or NF-kB prevented Tregs elimination and increased the population of Th1 and Th17 cells after nerve xenografting. Recent studies suggested that TLR ligands regulate $T$ cell activation, as well as $\mathrm{T}$ cell differentiation $[17,19]$. Therefore, TLR2 ligands may modulate $\mathrm{T}$ cell population through NF-KB by direct action because TLR2 are present on Tregs and Th cells. TLR2 was reported play a role in Tregs proliferation, and activation of TLR2 can cause increased activity of Tregs [20, 21]. In addition, reduction of Tregs was observed in TLR2-/- mice [22]. However, TLR2 also promotes Th1 and Th17 cell polarization [23]. Activation of TLRs also activates effector Th cells to enhance Th cell-mediated cytotoxicity and result in transplantation failure [8, 20]. Therefore, the graft immune tolerance and transplantation success may correlate with the balance of Treg/Th cells $[8,24]$.

The TLR2-coupled protein, MyD88, is reported to be important for Th17 immunity against allogeneic grafts [25]. A similar study also suggested that TLR2 signaling leads to Th1 immunity [26]. Although a previous study suggested that TLR2 activation promotes the Th2 immune response [27], knock-out of 
TLR2 altered Th1 but not Th2 population after nerve xenografting in this study. According to the Th1/Th2 balance hypothesis [10], the TLR2-NF-kB signaling pathway might have influenced the Th1-driven immune response in our nerve xenograft animal model.

Th17 cells, a newly recognized distinct subset of $\mathrm{T}$ helper cells, have been shown to play an important role in murine autoimmune diseases. They express a variety of potent proinflammatory cytokines in several autoimmune states [28]. Stimulation of TLR-induced NF-KB activation promotes differentiation of activated T cells into Th1 and Th17 cells [29]. Th1 and Th17 cells both activate in response to tissue transplantation to produce cytotoxicity [2]. Our results demonstrated that decrease of the Th1 and Th17 population in nerve xenografting in the TLR2 $\%$ or NF-kB-/- mice, implying that reduction of Th1 and Th17 population may ameliorate tissue damage after nerve transplantation.

\section{Conclusions}

In conclusion, our results suggest that TLR2 and its downstream target NF- $\mathrm{kB}$ are important in immune homeostasis. Knock-out of TLR2 or NF-kB prevented Tregs elimination and inhibited Th1 and Th17 populations increase, and thus may impair Th1and Th17-driven immune response after nerve xenografting. TLR2 inhibitors may further provide a potential prevention strategy for innate immunity-mediated graft rejection.

\section{Acknowledgements}

The work was supported by Chang Gung Memorial Hospital (CMRPG8A0022 \& CMRPG8A0023) to Ching-Hua Hsieh.

\section{Competing Interests}

The authors have declared that no competing interest exists.

\section{References}

[1] Andrade CF, Waddell TK, Keshavjee S, Liu M. Innate immunity and organ transplantation: the potential role of toll-like receptors. American journal of transplantation : official journal of the American Society of Transplantation and the American Society of Transplant Surgeons. 2005;5:969-75.

[2] Porrett PM, Yuan X, LaRosa DF, Walsh PT, Yang J, Gao W, et al. Mechanisms underlying blockade of allograft acceptance by TLR ligands. Journal of immunology. 2008;181:1692-9.

[3] Ivashkiv LB. A signal-switch hypothesis for cross-regulation of cytokine and TLR signalling pathways. Nature reviews Immunology. 2008;8:816-22.

[4] Kwok SK, Cho ML, Her YM, Oh HJ, Park MK, Lee SY, et al. TLR2 ligation induces the production of IL-23/IL-17 via IL-6, STAT3 and NF-kB pathway in patients with primary Sjogren's syndrome. Arthritis research \& therapy. 2012;14:R64.

[5] Zhang P, Liu X, Li Y, Zhu X, Zhan Z, Meng J, et al. Protein tyrosine phosphatase with proline-glutamine-serine-threonine-rich motifs negatively regulates TLR-triggered innate responses by selectively inhibiting IkappaB kinase beta/NF-kappaB activation. Journal of immunology. 2013;190:1685-94

[6] Vignali DA, Collison LW, Workman CJ. How regulatory T cells work. Nature reviews Immunology. 2008:8:523-32.
[7] Wolf D, Schreiber TH, Tryphonopoulos P, Li S, Tzakis AG, Ruiz P, et al. Tregs expanded in vivo by TNFRSF 25 agonists promote cardiac allograft survival. Transplantation. 2012;94:569-74.

[8] Sagoo P, Lombardi G, Lechler RI. Relevance of regulatory T cell promotion of donor-specific tolerance in solid organ transplantation. Frontiers in immunology. 2012;3:184.

[9] Shalev I, Selzner N, Shyu W, Grant D, Levy G. Role of regulatory T cells in the promotion of transplant tolerance. Liver transplantation : official publication of the American Association for the Study of Liver Diseases and the International Liver Transplantation Society. 2012;18:761-70.

[10] Kidd P. Th1/Th2 balance: the hypothesis, its limitations, and implications for health and disease. Alternative medicine review : a journal of clinical therapeutic. 2003;8:223-46.

[11] Chen Y, Wood KJ. Interleukin-23 and TH17 cells in transplantation immunity: does 23+17 equal rejection? Transplantation. 2007;84:1071-4.

[12] Zhang X, Beduhn M, Zheng X, Lian D, Chen D, Li R, et al. Induction of alloimmune tolerance in heart transplantation through gene silencing of TLR adaptors. American journal of transplantation : official journal of the American Society of Transplantation and the American Society of Transplant Surgeons. 2012;12:2675-88.

[13] Mandapathil M, Hilldorfer B, Szczepanski MJ, Czystowska M, Szajnik M, Ren $\mathrm{J}$, et al. Generation and accumulation of immunosuppressive adenosine by human CD4+CD25highFOXP3+ regulatory T cells. The Journal of biological chemistry. 2010;285:7176-86.

[14] Schmidt A, Oberle N, Krammer PH. Molecular mechanisms of treg-mediated T cell suppression. Frontiers in immunology. 2012;3:51.

[15] Hanidziar D, Koulmanda M. Inflammation and the balance of Treg and Th17 cells in transplant rejection and tolerance. Current opinion in organ transplantation. 2010;15:411-5.

[16] Zhang N, Kruger B, Lal G, Luan Y, Yadav A, Zang W, et al. Inhibition of TLR4 signaling prolongs Treg-dependent murine islet allograft survival. Immunology letters. 2010;127:119-25.

[17] Jin B, Sun T, Yu XH, Yang YX, Yeo AE. The effects of TLR activation on T-cell development and differentiation. Clinical \& developmental immunology. 2012;2012:836485.

[18] Land WG. Innate immunity-mediated allograft rejection and strategies to prevent it. Transplantation proceedings. 2007;39:667-72

[19] Goldstein DR, Palmer SM. Role of Toll-like receptor-driven innate immunity in thoracic organ transplantation. The Journal of heart and lung transplantation : the official publication of the International Society for Heart Transplantation. 2005;24:1721-9.

[20] Oberg HH, Juricke M, Kabelitz D, Wesch D. Regulation of T cell activation by TLR ligands. European journal of cell biology. 2011;90:582-92.

[21] Dai J, Liu B, Li Z. Regulatory T cells and Toll-like receptors: what is the missing link? International immunopharmacology. 2009;9:528-33.

[22] Sutmuller RP, den Brok MH, Kramer M, Bennink EJ, Toonen LW, Kullberg BJ, et al. Toll-like receptor 2 controls expansion and function of regulatory $\mathrm{T}$ cells. The Journal of clinical investigation. 2006;116:485-94.

[23] Aliahmadi E, Gramlich R, Grutzkau A, Hitzler M, Kruger M, Baumgrass R, et al. TLR2-activated human langerhans cells promote Th17 polarization via IL-1beta, TGF-beta and IL-23. European journal of immunology. 2009:39:1221-30.

[24] Yang J, Fan H, Hao J, Ren Y, Chen L, Li G, et al. Amelioration of acute graft-versus-host disease by adoptive transfer of ex vivo expanded human cord blood CD4+CD25+ forkhead box protein 3+ regulatory $\mathrm{T}$ cells is associated with the polarization of Treg/Th17 balance in a mouse model. Transfusion. 2012;52:1333-47.

[25] Wu H, Noordmans GA, O'Brien MR, Ma J, Zhao CY, Zhang GY, et al. Absence of MyD88 signaling induces donor-specific kidney allograft tolerance. Journal of the American Society of Nephrology : JASN. 2012;23:1701-16.

[26] Byun EH, Kim WS, Kim JS, Jung ID, Park YM, Kim HJ, et al. Mycobacterium tuberculosis Rv0577, a novel TLR2 agonist, induces maturation of dendritic cells and drives Th1 immune response. FASEB journal : official publication of the Federation of American Societies for Experimental Biology. 2012;26:2695-711.

[27] Redecke V, Hacker H, Datta SK, Fermin A, Pitha PM, Broide DH, et al. Cutting edge: activation of Toll-like receptor 2 induces a Th2 immune response and promotes experimental asthma. Journal of immunology. 2004;172:2739-43.

[28] Korn T, Oukka M, Kuchroo V, Bettelli E. Th17 cells: effector T cells with inflammatory properties. Seminars in immunology. 2007;19:362-71.

[29] Lobo PI, Bajwa A, Schlegel KH, Vengal J, Lee SJ, Huang L, et al. Natural IgM anti-leukocyte autoantibodies attenuate excess inflammation mediated by innate and adaptive immune mechanisms involving Th-17. Journal of immunology. 2012;188:1675-85. 